V. Vâjâitu

Nagoya Math. J.

Vol. 151 (1998), 99-106

\title{
ONE DIMENSIONAL FIBERING OVER $q$-COMPLETE SPACES
}

\author{
VIOREL VÂJÂITU
}

\begin{abstract}
We show that if $E \longrightarrow X$ is a locally trivial holomorphic fibrations whose fiber is an open Riemann surface and $X$ is a $q$-complete space, then $E$ is $q$-complete.
\end{abstract}

\section{$\S 1$. Introduction}

Let $X$ and $F$ be complex manifolds and $E$ a holomorphic fiber bundle over $X$ with typical fiber $F$. In 1953 Serre [7] posed the following question related to the classical Levi problem for characterizing domains of holomorphy:

$(\star)$ Assume that $X$ and $F$ are Stein. Does it follow that $E$ is Stein, too?

Several particular cases of this were settled (cf. [8] for summaries) until Skoda [9] produced in 1977 a counterexample with fiber $\mathbf{C}^{2}$, which, however, did not stop the interest around the problem. Mok [5] solved completely the case when $F$ is a complex curve. On the other hand, new questions have appeared, e.g., to study cohomological properties of such an $E$ as in ( $\star$. In this direction, Jennane [4] showed that the cohomology of $E$ with coefficients in coherent analytic sheaves is trivial in dimensions $\geq 2$. Furthermore, we established a general vanishing theorem for locally $q$-complete morphisms over $p$-complete spaces [12]. (The normalization is chosen so that Stein spaces correspond to 1-complete spaces.)

In this paper, by reconsidering the geometrical point of view, we extend Mok's result to the case $X$ is $q$-complete by proving the following theorem which answers a question raised to the author by Professor Takeo Ohsawa at the Conference on complex analysis in Hayama, Japan in the spring of 1995.

Received December 12, 1996. 
THEOREM 1. Let $\pi: E \rightarrow X$ be a locally trivial holomorphic fiber space with typical fiber $F$. Assume that $F$ is an open Riemann surface. If $X$ is $q$-complete, then $E$ is q-complete, too.

Note that for fibers of dimensions $\geq 2$ there are counterexamples [12]. In fact, for every integer $q>1$ there is a fiber bundle $E$ with fiber $\mathbf{C}^{2}$ over a $q$-complete domain in $\mathbf{C}^{q+1}$ such that $H^{q}\left(E, \mathcal{O}_{E}\right)$ does not vanish, a fortiori $E$ is not $q$-complete [1].

The method we use in proving Theorem 1 yields also the subsequent interesting criterion of $q$-completeness which was suggested to me by Professor Mihnea Colthoiu and may be viewed, in a weak sense, as a theorem of Docquier-Grauert type [3] for $q$-complete manifolds.

THEOREM 2. Let $X$ be a weakly q-complete complex space such that on every relatively compact open subset there are continuous strongly plurisubharmonic functions. Then $X$ is q-complete.

\section{§2. Preliminaries}

Throughout this paper all complex spaces are assumed to be reduced and with countable topology. By an open Riemann surface we mean a non-singular complex curve without compact components.

Let $X$ be a complex space and $T_{x} X$ denotes the (Zariski) tangent space of $X$ at $x$. Set $T X=\cup_{x \in X} T_{x} X$. If $X=\mathbf{C}^{n}, T_{x} X$ is canonically identified with $\mathbf{C}^{n}$.

A (local) chart of $X$ at a point $x \in X$ is a holomorphic embedding $\iota: U \rightarrow \widehat{U}$, where $U \ni x$ is an open subset of $X$ and $\widehat{U}$ is an open subset of some euclidean space $\mathbf{C}^{n}$. Holomorphic embedding means that $\iota(U)$ is an analytic subset of $\widehat{U}$ and the induced map $\iota: U \rightarrow \iota(U)$ is biholomorphic.

Suppose $\iota: U \rightarrow \widehat{U}$ is a local chart at $x$; then the differential map $\iota_{*}: T_{x} X \rightarrow \mathbf{C}^{n}$ of $\iota$ at $x$ is an injective homomorphism of complex vector spaces.

Let $D \subset \mathbf{C}^{n}$ be an open subset. A function $\varphi \in C^{\infty}(D, \mathbf{R})$ is said to be $q$-convex if the quadratic form

$$
L(\varphi, z)(\xi)=\sum_{i, j=1}^{n} \frac{\partial^{2} \varphi}{\partial z_{i} \partial \bar{z}_{j}}(z) \xi_{i} \bar{\xi}_{j}, \quad \xi \in \mathbf{C}^{n}
$$

has at least $n-q+1$ positive eigenvalues for every $z \in D$, or equivalently, there exists a family $\left\{M_{z}\right\}_{z \in D}$ of $(n-q+1)$-dimensional complex vector subspaces of $\mathbf{C}^{n}$ such that $L(\varphi, z)_{\mid M_{z}}$ is a positive definite form for all $z \in D$. 
Let $X$ be a complex space. A function $\varphi \in C^{\infty}(X, \mathbf{R})$ is said to be $q$-convex if every point of $X$ admits a local chart $\iota: U \rightarrow \widehat{U} \subset \mathbf{C}^{n}$ such that there is an extension $\widehat{\varphi} \in C^{\infty}(\widehat{U}, \mathbf{R})$ of $\varphi_{\mid U}$ which is $q$-convex on $\widehat{U}$. (This definition does not depend on the local embeddings.)

We say that $X$ is $q$-complete if there exists a $q$-convex exhaustion function $\varphi \in C^{\infty}(X, \mathbf{R})$.

A subset $\mathcal{M} \subset T X$ is said to be a linear set over $X$ (of codimension less than $q$ ) if for every point $x \in X, \mathcal{M}_{x}:=\mathcal{M} \cap T_{x} X \subset T_{x} X$ is a complex vector subspace (of codimension less than $q$ ). If $W \subset X$ is an open set, we have an obvious definition of the restriction $\mathcal{M}_{\mid W}$. The following is due to Peternell [6].

Definition. Let $X$ be a complex space, $W \subset X$ an open set, $\mathcal{M}$ a linear set over $W$, and $\varphi \in C^{\infty}(W, \mathbf{R})$.

(a) Let $x \in W$. Then we say that $\varphi$ is weakly 1 -convex with respect to $\mathcal{M}_{x}$ if there are: a local chart $\iota: U \rightarrow \widehat{U}$ of $X$ with $x \in U \subset W, \widehat{U} \subset \mathbf{C}^{n}$ open set, and an extension $\widehat{\varphi} \in C^{\infty}(\widehat{U}, \mathbf{R})$ of $\varphi_{\mid U}$ such that $L(\widehat{\varphi}, \iota(x))\left(\iota_{*} \xi\right) \geq 0$ for every $\xi \in \mathcal{M}_{x}$.

We say that $\varphi$ is weakly 1 -convex with respect to $\mathcal{M}$ if $\varphi$ is weakly 1-convex with respect to $\mathcal{M}_{x}$ for every $x \in W$.

(b) The function $\varphi$ is said to be 1-convex with respect to $\mathcal{M}$ if every point of $W$ admits an open neighborhood $U \subset W$ such that there exists a 1-convex function $\theta$ on $U$ with $\varphi-\theta$ weakly 1-convex with respect to $\mathcal{M}_{\mid U}$.

It is not difficult to see that the extension $\widehat{\varphi}$ of $\varphi$ is irrelevant for the above definition. In particular, if the functions $\varphi$ and $\psi$ are (weakly) 1convex with respect to $\mathcal{M}$, so is their $\operatorname{sum} \varphi+\psi$.

On the other hand, a complex space $X$ is $q$-complete if, and only if, there exists a linear set $\mathcal{M}$ over $X$ of codimension less than $q$ and an exhaustion function $\varphi \in C^{\infty}(X, \mathbf{R})$ which is 1-convex with respect to $\mathcal{M}$.

Motivated by this observation, we call a complex space $X$ weakly $q$ complete if there exists a linear set $\mathcal{M}$ over $X$ of codimension less than $q$ and an exhaustion function $\varphi \in C^{\infty}(X, \mathbf{R})$ which is weakly 1-convex with respect to $\mathcal{M}$.

Here, to avoid some technical difficulties in the proofs of the theorems, we introduce the following

Definition. Let $X$ be a complex space and $\mathcal{M}$ a linear set over $X$. We say that $\varphi \in C^{0}(X, \mathbf{R})$ is (weakly) $\mathcal{M}$-convex if, and only if, every point of $X$ 
admits an open neighborhood $D$ such that there are functions $f_{1}, \ldots, f_{k} \in$ $C^{\infty}(D, \mathbf{R})$, (weakly) 1 -convex with respect to $\mathcal{M}$, and $g_{1}, \ldots, g_{k} \in \operatorname{Psh}(D) \cap$ $C^{0}(D, \mathbf{R})$ with

$$
\varphi_{\mid D}=\max \left\{f_{1}+g_{1}, \ldots, f_{k}+g_{k}\right\} .
$$

For instance, if $\varphi$ is weakly $\mathcal{M}$-convex and $\psi$ is $\mathcal{M}$-convex, then their sum $\varphi+\psi$ is $\mathcal{M}$-convex. Besides, if $h: \mathbf{R} \rightarrow \mathbf{R}$ is given locally by $\max \left\{a_{1} t+\right.$ $\left.b_{1}, \ldots, a_{k} t+b_{k}\right\}$ with $a_{i}>0$ for all $i$, then $h(\varphi)$ is again weakly $\mathcal{M}$-convex.

Remark. In [11] we introduced continuous functions $\varphi: X \rightarrow \mathbf{R}$ which we called pseudoconvex with respect to $\mathcal{M}$, i.e., one has (\$) without $g_{i}$ 's. Clearly, this notion is stronger than $\mathcal{M}$-convexity.

Therefore, the following approximation result improves [11], Theorem 1.

Proposition 1. Let $\mathcal{M}$ be a linear set over a complex space $X$ and $\varphi \in C^{0}(X, \mathbf{R})$ a $\mathcal{M}$-convex function. Then for every $\eta \in C^{0}(X, \mathbf{R}), \eta>0$, there exists $\widetilde{\varphi} \in C^{\infty}(X, \mathbf{R})$ which is 1-convex with respect to $\mathcal{M}$ and

$$
\varphi \leq \widetilde{\varphi}<\varphi+\eta \text {. }
$$

In particular, if $\mathcal{M}$ has codimension less than $q$, then $\tilde{\varphi}$ is $q$-convex.

Proof. Choose a locally finite covering $\left\{U_{i}\right\}_{i \in I}$ of $X$ by relatively compact open Stein subsets on which there are functions $f_{i j}, g_{i j}, j=1, \ldots, n_{i}$, as in (\$). Then consider open sets $V_{i} \subset \subset U_{i}$ and compact sets $K_{i} \subset V_{i}$ such that $\left\{K_{i}\right\}_{i \in I}$ is again a covering of $X$. Let $\theta_{i}$ be smooth functions on $X$ which equal -1 on $\partial V_{i}$ and 1 on $K_{i}$. Choose $\delta_{i}>0$ small enough such that $f_{i j}^{\prime}:=\delta_{i} \theta_{i}+f_{i j}, j=1, \ldots, n_{i}$, are 1 -convex with respect to $\mathcal{M}$ on $V_{i}$, and $2 \delta_{i}<\inf _{V_{i}} \eta$ for all $i$. Then choose smooth plurisubharmonic functions $g_{i j}^{\prime}$ on $U_{i}$ such that $\left|g_{i j}^{\prime}-g_{i j}\right|<\delta_{i}$ on $V_{i}$. One may define $\varphi^{\prime}: X \rightarrow \mathbf{R}$ by: for every $x \in X$ set

$$
\varphi^{\prime}(x)=\sup \left\{f_{i j}^{\prime}(x)+g_{i j}^{\prime}(x) ; i, j \text { such that } x \in V_{i}\right\} .
$$

It is straightforward to see that $\varphi^{\prime}$ is continuous, 1-convex with respect to $\mathcal{M}$ on $X$, and $\varphi<\varphi^{\prime}<\varphi+\eta$. The proposition follows now by [11], Theorem 1 , if we approximate $\varphi^{\prime}$ in the $C^{0}$-topology by $\tilde{\varphi}$ as required.

The subsequent is proved in [5].

THEOREM 3. If $S$ is a connected open Riemann surface, then there exists an exhaustion function $\varphi \in \operatorname{Psh}(S) \cap C^{0}(S, \mathbf{R})$ such that for every automorphism $\theta$ of $S, \varphi-\varphi \circ \theta$ is a bounded function. 


\section{§3. The proofs of the theorems}

To begin with, we state a proposition, which, by taking into account the definition of a weakly $q$-complete space, gives immediately a proof of Theorem 2.

Proposition 2. Let $\mathcal{M}$ be a linear set over a complex space $X$. Suppose that:

a) There exists an exhaustion function $\varphi \in C^{0}(X, \mathbf{R})$ which is weakly $\mathcal{M}$-convex.

b) For every open set $D \subset \subset X$ there exists $\psi \in C^{\infty}(X, \mathbf{R})$ which is 1-convex with respect to $\mathcal{M}$ on $D$.

Then there exists an exhaustion function $\tilde{\varphi} \in C^{\infty}(X, \mathbf{R})$ which is 1 convex with respect to $\mathcal{M}$ on $X$.

Proof. Without any loss of generality, we may assume that $\min _{X} \varphi=0$. For $n=1,2, \ldots$, we denote $K_{n}=\{x \in X ; \varphi(x) \leq n\}$ and $D_{n}=\{x \in$ $X ; \varphi(x)<n+2\}$. Let $\psi_{n} \in C^{\infty}(X, \mathbf{R})$ be $\mathcal{M}$-convex on $D_{n+1}$. By taking the exponential, we may suppose $\psi_{n}>0$. Set

$$
a_{n}:=\max _{K_{n+2}}\left(\varphi+\psi_{n}\right)>0 .
$$

Let $h_{n}: \mathbf{R} \rightarrow \mathbf{R}$ be defined by $h_{n}(t)=\max \left\{t,\left(1+a_{n}\right)(t-n-1)\right\}, t \in \mathbf{R}$. Clearly, $h_{n}$ is strictly increasing, convex, $h_{n}(t)=t$ for $t \leq n+1$, and $h_{n}(n+2)>a_{n}$. Therefore $h_{n}(\varphi)>\varphi+\psi_{n}$ on the set $\{n+2-\varepsilon<\varphi<n+2\}$ for some $\varepsilon>0$ sufficiently small; consequently we may define a continuous function $\varphi_{n}: X \rightarrow \mathbf{R}$ by:

$$
\varphi_{n}=\left\{\begin{array}{lll}
\max \left(\varphi+\psi_{n}, h_{n}(\varphi)\right) & \text { on } & D_{n} \\
h_{n}(\varphi) & \text { on } & X \backslash D_{n} .
\end{array}\right.
$$

Then $\varphi_{n}$ is positive and exhaustive since $\varphi_{n} \geq h_{n}(\varphi)$, weakly $\mathcal{M}$-convex on $X$, and as $\varphi_{n}=\varphi+\psi_{n}$ on $K_{n+1}, \varphi_{n}$ is a $\mathcal{M}$-convex function on the interior of $K_{n+1}$.

Now, if the sequence $\left\{\epsilon_{n}\right\}_{n}$ of positive numbers decreases (fast enough) to 0 , we may define an exhaustion $\mathcal{M}$-convex function $\Phi \in C^{0}(X, \mathbf{R})$ by

$$
\Phi=\varphi+\sum \varepsilon_{n} \varphi_{n}
$$

and we conclude by Proposition 1 . 
Here we start the proof of Theorem 1. First assume that the fiber $F$ is connected (for steps 1 and 2); second, the general case (step 3), will follow by a canonical reduction procedure if we note some facts on $q$-complete spaces.

Step 1. By Theorem 3 and standard arguments, there exists a locally finite covering $\left\{U_{i}\right\}_{i \in I}$ of $X$ by relatively compact open Stein subsets which trivialize $E$ and such that the following property holds:

(•) There are continuous plurisubharmonic exhaustion functions $\varphi_{i}: E_{i} \rightarrow$ $\mathbf{R}, E_{i}=\pi^{-1}\left(U_{i}\right)$, such that for every compact set $K \subset U_{i} \cap U_{j}, \varphi_{i}-\varphi_{j}$ are bounded functions on $\pi^{-1}(K)$ for every indices $i$ and $j$. Clearly, we may suppose $\varphi_{i}>0$.

Now consider open sets $W_{i} \subset \subset V_{i} \subset \subset U_{i}$ such that $\cup W_{i}=X$; then choose non-negative smooth functions $p_{i}^{\prime}$ with compact support contained in $V_{i}$ which equal 1 on $W_{i}$. Since on $\pi^{-1}\left(W_{i} \cap \partial V_{j}\right)$ one has: $\varphi_{i}-\varphi_{j}$ is a bounded function, $p_{i}^{\prime} \circ \pi=1$, and $p_{j}^{\prime} \circ \pi=0$, there are large enough constants $C_{i}>0$ such that on $\pi^{-1}\left(W_{i} \cap \partial V_{j}\right)$ one has:

$$
C_{i} p_{i}^{\prime}+\varphi_{i}>\varphi_{j}=C_{j} p_{j}^{\prime}+\varphi_{j}
$$

for all indices $i$ and $j$. Put $p_{i}:=C_{i} p_{i}^{\prime}, i \in I$, and define $u: E \rightarrow(0, \infty)$ by

$$
u(\zeta)=\max \left\{p_{i}(\pi(\zeta))+\varphi_{i}(\zeta) ; i \in I(\zeta)\right\}
$$

where for $\zeta \in E, I(\zeta):=\left\{i \in I ; \pi(\zeta) \in V_{i}\right\}$. One checks readily that $u$ is continuous and for an arbitrary compact set $L \subset X$ the restriction of $u$ to $\pi^{-1}(L)$ is exhaustive.

Step 2. Let $\mathcal{M}=\left\{\mathcal{M}_{x}\right\}_{x \in X}$ be a linear set over $X$ of codimension less than $q$ and $\varphi^{\prime} \in C^{\infty}(X, \mathbf{R})$ an exhaustion function which is 1-convex with respect to $\mathcal{M}$. Select a smooth non-negative function $\lambda$ from $\mathbf{R}$ into itself which is rapidly increasing and convex such that $\lambda\left(\varphi^{\prime}\right)+p_{i}, i \in I$, are 1-convex with respect to $\mathcal{M}$. Set $\varphi:=\lambda\left(\varphi^{\prime}\right)$.

Now define a linear set $\mathcal{N}$ over $E$ by $\mathcal{N}_{\zeta}=\pi_{*, \zeta}^{-1}\left(\mathcal{M}_{\pi(\zeta)}\right)$ for $\zeta \in E$. Here $\pi_{*, \zeta}$ means the differential map of $\pi$ at $\zeta$ from $T_{\zeta} E$ into $T_{\pi(\zeta)} X$. Obviously, $\mathcal{N}$ has codimension less than $q$.

Put $\sigma=\varphi \circ \pi+u$. Then $\sigma$ is weakly $\mathcal{N}$-convex on $E$, and by what we said in Step 1, $\sigma$ is exhaustive. 
We claim that for every open set $\Omega \subset \subset E$ there exists a smooth function $\psi$ on $E$ which is 1-convex with respect to $\mathcal{N}$ on $\Omega$.

Indeed, we cover $\overline{\pi(\Omega)}$ with finitely many $W_{i}$ 's, say $W_{i}, i=1, \ldots, m$; then choose positive 1-convex functions $\psi_{i}$ on $\pi^{-1}\left(U_{i}\right)$. Straightforward computations show that there exists a constant $A_{0}>0$ large enough such that the function $\psi_{A} \in C^{\infty}(E, \mathbf{R})$ given by

$$
\psi_{A}=A \cdot(\varphi \circ \pi)+\sum_{i+1}^{m}\left(p_{i} \circ \pi\right) \cdot \psi_{i}
$$

is 1 -convex with respect to $\mathcal{N}$ on $\Omega$ for every $A \geq A_{0}$. Now we conclude the proof of the theorem by applying Proposition 2 .

Step 3. Here we consider the general case. In order to do this, decompose $F=\cup F_{j}$ so that in $F_{j}$ appear only connected components isomorphic to each other and non-isomorphic to connected components of $F_{s}$, for $s \neq j$. Each $F_{j}$ is invariant under transition automorphisms of $E$, so that $E$ splits into a disjoint union of fiber bundles $E_{j}$ with base $X$ and fiber $F_{j}$. Thus it suffices to assume that the fiber $F$ consists of isomorphic connected components. Then the transition automorphisms can permute the connected components of $F$ and we have a two-step fibration $E \rightarrow \widetilde{X} \rightarrow X$ where $\widetilde{X}$ is a topological covering of $X$ and the first fibration has a connected fiber. Since $\widetilde{X}$ is $q$-complete by [2], the theorem follows now from the preceding case.

Acknowledgements. I thank the DFG for financial support and Professor Klas Diederich for his hospitality allowing me to complete this paper at the university of Wuppertal. Also I thank the referee for some useful comments on a preliminary version of this paper.

\section{REFERENCES}

[1] A. Andreotti and H. Grauert, Théorèmes de finitude pour la cohomologie des espaces complexes, Bull. Soc. Math. France, 90 (1962), 193-259.

[2] E. Ballico, Coverings of complex spaces and q-completeness, Riv. Mat. Univ. Parma (4), 7 (1981), 443-452.

[3] F. Docquier and H. Grauert, Levisches Problem und Rungescher Satz für Teilgebiete Steinscher Mannigfaltigkeiten, Math. Ann., 140 (1960), 94-123.

[4] B. Jennane, Groupes de cohomologie d'un fibre holomorphe a base et a fibre de Stein, Invent. Math., 54 (1979), 75-79. 
[5] N. Mok, The Serre problem on Riemann surfaces, Math. Ann., 258 (1981), 145-168.

[6] M. Peternell, Algebraische Varietäten und q-vollständige komplexe Räume, Math. Z., 200 (1989), 547-581.

[7] J. P. Serre, Quelques problèmes globaux relatifs aux variétés de Stein, Colloque sur les fonctions de plusieurs varibales, Bruxelles 1953, 53-68.

[8] Y.-T. Siu, Pseudoconvexity and the problem of Levi, Bull. Amer. Math. Soc., 84 (1978), 481-511.

[9] H. Skoda, Fibrés holomorphes à base et à fibre de Stein, Invent. Math., 43 (1977), 97-107.

[10] J.-L. Stehlé, Fonctions plurisousharmoniques et convexité holomorphe de certain fibrés analytiques, Lecture Notes, 474, Séminaire P. Lelong, 1973/74, 155-180.

[11] V. Vâjâitu, Approximation theorems and homology of $q$-Runge domains in complex spaces, J. reine angew. Math., 449 (1994), 179-199.

[12] Cohomology groups of locally q-complete morphisms with p-complete base, Math. Scandinavica, 79 (1996), 161-175.

Institute of Mathematics of the Romanian Academy

P.O.Box 1-764, RO-70700 Bucharest

Romania

vvajaitu@stoilow.imar.ro 